\title{
Investigation of fNIRS Brain Sensing as Input to Information Filtering Systems
}

\author{
Evan M. Peck \\ Tufts University \\ evan.peck@tufts.edu
}

\author{
Daniel Afergan \\ Tufts University \\ afergan@cs.tufts.edu
}

\author{
Robert J.K. Jacob \\ Tufts University \\ jacob@cs.tufts.edu
}

\begin{abstract}
Today's users interact with an increasing amount of information, demanding a similar increase in attention and cognition. To help cope with information overload, recommendation engines direct users' attention to content that is most relevant to them. We suggest that functional near-infrared spectroscopy (fNIRS) brain measures can be used as an additional channel to information filtering systems. Using fNIRS, we acquire an implicit measure that correlates with user preference, thus avoiding the cognitive interruption that accompanies explicit preference ratings. We explore the use of fNIRS in information filtering systems by building and evaluating a brain-computer movie recommender. We find that our system recommends movies that are rated higher than in a control condition, improves recommendations with increased interaction with the system, and provides recommendations that are unique to each individual.
\end{abstract}

\section{Categories and Subject Descriptors}

H.5.2 [Information Interfaces and Presentation:]: User Interfaces

\section{General Terms}

Human Factors: Design; Measurement.

\section{Keywords}

fNIRS, information filtering, brain-computer interfaces, passive input, adaptive interfaces

\section{INTRODUCTION}

User attention is a scarce resource in modern computing. Mental resources are often divided among disparate but concurrent streams of information. In the wake of such pervasive distractions, research has shown that focusing on the wrong information or consuming information at the wrong moment can not only lead to a decrease in performance during work, but negatively impact work satisfaction, and increase stress and anxiety $[2,16,26]$.

Permission to make digital or hard copies of all or part of this work for personal or classroom use is granted without fee provided that copies are not made or distributed for profit or commercial advantage and that copies bear this notice and the full citation on the first page. To copy otherwise, or republish, to post on servers or to redistribute to lists, requires prior specific permission and/or a fee.

AH '13, March 07-08 2013, Stuttgart, Germany

Copyright 2013 ACM 978-1-4503-1904-1/13/03 \$15.00.
To address some of these problems, researchers have suggested that physiological measures of workload or attention should be used to deliver information at an opportune time. For example, Bailey et al. used pupil dilation as a measure of workload for interruption [3], and Solovey et al. built an interactive system that adapts robot automation to a human operator's working memory load [34]. However, while physiological computing has been used to manipulate when information is delivered to the user, very little work has focused on which information should be delivered.

In this paper, we explore the use of functional nearinfrared spectroscopy (fNIRS) brain sensing to classify preference judgments and drive information filtering systems. fNIRS has recently gained traction in the HCI community because it is generally resistant to movement artifacts [20] and observes physiological parameters that are inaccessible to other brain and body sensors [36, 38]. Given recent neuroscience literature, these parameters may allow the detection of preference judgments that extend beyond emotional response by incorporating the reasoning processes of the brain [5, 25]. Thus, if there is any correlation between fNIRS signals and preference judgments, fNIRS could potentially augment current practices by being used as an additional source of passive information to filtering systems.

However, there are significant challenges to the use of fNIRS in information filtering systems. Previous fNIRS work has analyzed preference judgments exclusively in offline environments [25]. Additionally, signals that correlate with preference are often subtle and may not translate to real world use cases. For these reasons, a primary goal of this paper is to explore whether fNIRS preference measures can be used in a real-time environment.

To investigate the use of fNIRS in information filtering systems, we present an automated recommendation system that suggests new movies based on fNIRS measures alone. Using fNIRS to monitor the prefrontal cortex, our system classifies brain data in real-time and iteratively updates a model of user preference to recommend movies that are personalized to the individual user. To evaluate our system, we ran a user study and found that fNIRS can contribute information to a recommendation environment by outperforming a no-input control condition. In addition, we observed that the system's model of user preference improved the longer the user interacted with the de- 
vice. Finally, we found that recommendations were uniquely catered to the individual - $45 \%$ of the movies each participant viewed were not recommended to any other participant - showing that we were responding to individual preference and not overall popularity.

We suggest that this brain recommendation system acts as a proof-of-concept for the use of fNIRS as input to information filtering systems. We argue that eventually by integrating when information should be delivered along with which information should be emphasized, brain-computer interfaces have the potential to automatically provide users with the right information at the right moment.

We make the following contributions: We show that fNIRS brain sensing can be used as input to information filtering systems. We construct and evaluate a real-time movie recommendation system that is driven by brain signals that correlate with preference. We find that our system recommends higher-rated movies with fNIRS input than without it, and that the underlying model of user preference improves over time. Second, we discuss the implications of using fNIRS measures in information filtering systems. We suggest that brain sensing can someday augment current recommendation systems, support the creation of recommendation systems in new domains, and unify recommendations across disparate information sources.

\section{BACKGROUND AND RELATED WORK}

In this work, we focus primarily on preference judgments as a key input to information filtering. When integrated with information filtering algorithms, preference enables users to allocate attention through recommendations of personalized information or products [33]. Thus, preference helps determine which information should be presented to the user. In addition, preference has been integrated into applications such as personal search [37], prioritizing incoming text and voice messages [27], optimizing user interfaces [13], calculating interruption costs [21], and guiding conceptual design [4], among others.

In current information filtering systems, eliciting preference involves a tradeoff between accuracy and obtrusiveness. Explicit measures require users to record their own preferences through a rating scale. While explicit ratings are generally accurate representations of what the user prefers, they can act as attention-sinks by disturbing normal behavior with an interface and introducing an introspective cognitive step [30]. Additionally, responses depend on the assumption that users can sense and externalize their subjective emotions, which may not be true [22].

Implicit measures predict user preference by observing the user during natural interactions with a system, and are based on viewing history, purchase history, view time, or other behavioral measures $[8,12,29,31]$. These ratings are essentially elicited for free as they require no additional effort on the part of the user. However, implicit ratings are widely considered to be less accurate than explicit ratings because they are based on prediction models that might not reflect the user's preference and can be affected by a number of other variables [30]. For that reason, new methods are often proposed to increase the accuracy of implicit ratings.

\subsection{Physiological Measures of Preference}

One approach to increase the effectiveness of implicit measures is to incorporate physiological sensors into preference prediction models. For example, combinations of galvanic skin response (GSR), electromyograms (EMG), blood pressure, respiration pattern, and electroencephalography (EEG) have been used to capture emotional responses to videos $[6$, 23]. Following this work, there have been several attempts to use affective signals as input to recommendation engines. For example, Healey et al. [18] constructed an "Affective DJ" that dynamically constructed "energizing" or "relaxing" musical play lists. Similarly, Wu et al. [39] built a system that recommends multimedia with similar emotional content. In each of these cases, recommendations were grounded in emotional responses to content, and analysis of each system was largely preliminary.

\subsection{Preference Judgments in the Brain}

While preference judgments and emotional reactions are often linked, previous work indicates that there are two separate processing chains that combine to influence preference judgments: emotion and reason [5]. This is because preference (and specifically, economic decisions) may be based on various competing factors, such as price, usefulness, branding, and availability. For example, viewing a high-end sports car may elicit positive emotions, but a small, fuel-efficient hybrid car may elicit higher preference values.

The prefrontal cortex ( $P F C$ ) - a region of the brain situated just behind the forehead - has been identified as playing a critical role in the integration of emotional experience with reason in the decision-making process [10]. Thus, the PFC offers information about preference judgments that physiological sensors focusing on affective state may not detect. We ground our measures of preference in several studies that investigate the neural correlates of preference using functional magnetic resonance imaging (fMRI) and positron emission tomography (PET). In a study by Deppe et al., fMRI showed increased activation in the prefrontal cortex during economic decisions involving a preferred brand name [10]. Paulus et al. recorded similar results in preference judgments of drink categories [32]. Finally, McClure et al. compared activation in a blind Coke v. Pepsi test, finding that neural responses in the PFC were consistent with behavior [28].

\subsection{Using fNIRS to Detect Preference}

fNIRS is an optical brain sensing technology that has seen increased use in HCI research, largely because of its simple setup and resistance to movement artifacts $[1,15,19$, $34,35]$. Using near-infrared light, fNIRS measures localized levels of oxygenated and deoxygenated hemoglobin in the brain, successfully replicating results from fMRI studies $[36,38]$. However, the physiological response of sending oxygen to the brain takes 5-7 seconds after activation. This means that fNIRS is better suited for observing long-term user states, rather than instantaneous events. Given these features, fNIRS is seen as a technology that offers information that is complementary to other physiological measures.

In this paper, we apply fNIRS to the detection of preference in the PFC, observing similar physiological parameters to the fMRI studies noted above. Additionally, we base our research on a recent study by Luu and Chau (extending Paulus 


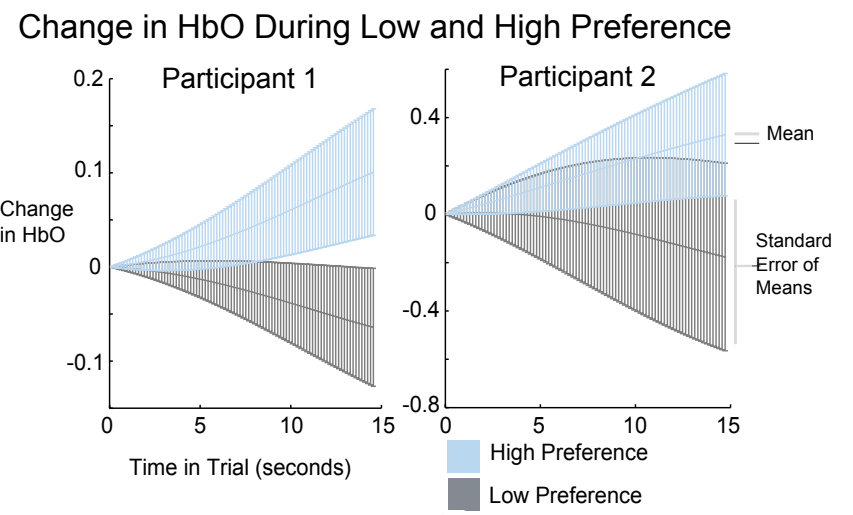

Figure 1: From a pilot study, an example of the fNIRS signal from the right hemisphere during periods of high preference and low preference. The plots show the mean change in oxygenated hemoglobin during 8 trials of high preference and 8 trials of low preference.

et al. [32]), where fNIRS measures of the PFC were used to classify extreme preferences for various beverage categories (e.g. milk, soda, water) [25]. In a pilot study of our own, we confirmed the results of Luu and Chau by observing different activation patterns in participants as they viewed pictures of digital music devices that they liked and disliked (Figure 1).

\subsection{Physiological Input to Adaptive Systems}

A key feature of our brain-recommender system is that it monitors preference without any specific effort from the user. The system reads passive information about users during natural interaction, and then adapts to their current state. While most work in BCI has focused on active brain-computer communication, more recent research has suggested the use of implicit neural parameters as input to adaptive systems $[9,11,15,40]$.

George and Lecuyer survey current passive BCI literature and categorize them into four application areas: 1) adapting the level of automation, 2) implicit multimedia content tagging, 3) video games, and 4) error correction and detection [14]. Although our work is most closely related to implicit multimedia content tagging, it extends that research by adding an adaptive element (new recommendations).

\section{THE BRAIN RECOMMENDER}

In order to explore whether fNIRS can provide useful input to information filtering systems, we constructed a movie recommender that is driven exclusively by fNIRS signals and compared it to a system that does not include passive user input (a no-recommendation environment). By controlling for behavioral indicators of preference, we test the ability of fNIRS to add information to recommendation systems beyond traditional behavioral metrics of viewing time and history. Thus, if a brain-driven recommender provides intelligent recommendations, we believe that implicit fNIRS measurements can be used to augment current techniques.

- Hypothesis: Observing the brain with fNIRS will allow the brain recommender system to construct a pref- erence model of the user, suggesting movies that cater to each user's interests.

In the following sections, we discuss the technical details of our system, report our experimental methods, and analyze the data from our experiment. Finally, we discuss the implications of our results, outlining a vision for a information filtering systems that are driven by the brain.

\section{SYSTEM DETAILS}

Constructing a fully-functional recommendation system based on brain input requires the coordination of a number of technological pieces. To provide a technical overview, we refer to Figure 3 and briefly discuss the flow of information in our system.

First, light sensing data is sent from our fNIRS data acquisition software to an analysis program built in our lab, where the signal is filtered to remove noise and movement artifacts. There, we partition the fNIRS data into segments of identical length to training examples we provided during an earlier training period. These segments are sent to Weka, an open-source machine learning library, where we classify the fNIRS signal based on previous examples [17]. This classification is sent to our Java application that holds the movie and rating database and serves as the backbone of our recommendation model. The application updates the database and recommendation model with new user information, and searches for the top recommended movie given all other previous data about the user. Finally, the selected recommendation is sent to a browser that navigates to the movie's corresponding IMDB page.

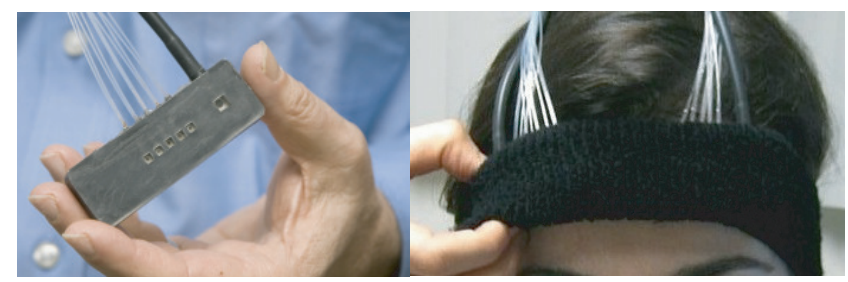

Figure 2: (Left) An fNIRS probe with four light sources and one detector. (Right) A researcher secures two fNIRS probes on a volunteer's forehead. This arrangement is used to measure the prefrontal cortex (PFC)

\subsection{Measuring and Filtering the fNIRS Signal}

We used a multichannel frequency domain OxiplexTX from ISS Inc. (Champaign, IL) for data acquisition. Two fNIRS probes were places on the forehead in order to measure the two hemispheres of the anterior prefrontal cortex (Figure 2 ). The source-detector distances were 1.5, 2, 2.5, and 3 $\mathrm{cm}$. Each distance measures a difference depth in the cortex. Each source emits two light wavelengths $(690 \mathrm{~nm}$ and $830 \mathrm{~nm}$ ) to detect and differentiate between oxygenated and deoxygenated hemoglobin. The sampling rate was $6.25 \mathrm{~Hz}$.

In order to remove noise that might be the result of user movement, respiration, or heart beats, we apply filtering techniques described by Solovey et al. in their adaptive system that also used fNIRS input [34]. First, we used an 


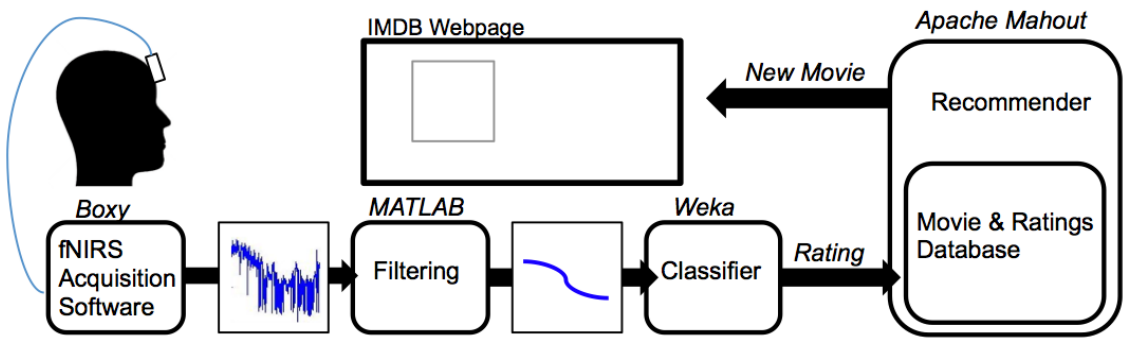

Figure 3: The basic architecture of our real-time classification system.

elliptic low pass filter with a cutoff frequency of $0.025 \mathrm{~Hz}$, stoppage frequency of $0.03 \mathrm{~Hz}$, max ripple of $3 \mathrm{~dB}$ and a stop band attenuation of $50 \mathrm{~dB}$. Next, we used a z-score to normalize the data in each information channel. Finally, for each training example, we calculated the signal change of each time point from the first time point in the example.

\subsection{Building the fNIRS Classifier}

Once each of the filtering steps was completed, we built a new preference model for each participant based on the training protocol we describe in the experiment section of this paper. We constructed a classifier that differentiated between low and high preference for each of the 16 information channels on our fNIRS device ( 2 probes $\mathrm{x} 4$ distances $\mathrm{x}$ 2 wavelengths), using the filtered light readings at each time point of a trial as individual features to the classifer. Since we sampled data at $6.25 \mathrm{~Hz}$, a 25 second trial would consist of approximately 156 features. Finally, we used a built-in support vector machine (SVM) algorithm from Weka's sequential minimal optimization (SMO) package.

While previous fNIRS work [25] suggested that we could discriminate between periods of low and high preference, the movie dataset we used to ground our recommendations was based on a 1 to 5 star rating. This left us with a mapping problem. Recall that we built a separate classifier for each information channel of the fNIRS device. To map classifications of low v. high preference to a 5-point rating scale, we took a percentage vote from the classifiers. For example, if $80-100 \%$ of our information channels classified the incoming data as a period of high preference, we mapped this classification to a 5 star rating. If $60-80 \%$ of our information channels classified the data as a period of high preference, we mapped this classification to a 4 star rating.

This mapping is not ideal in a real-world scenario, as classification uncertainty is not equivalent to preference intensity. However, we use this approach to accommodate for the necessary time constraints of a normal experimental session. We suggest a more robust approach in the discussion section.

\subsection{Dataset and Recommendation Engine}

To build the movie recommendation engine, we used the HetRec 2011 MovieLens Data Set, an extension of MovieLens10M dataset [7]. Our movie recommendation engine was constructed using Apache Mahout, an open source machine learning library for Java that includes built-in col- laborative filtering algorithms. Our user recommendation system was based on a nearest neighbor algorithm using a similarity metric of euclidean distance.

\section{EXPERIMENT}

In order to evaluate the brain-computer recommender, we describe the experiment protocol in two sections: training and testing. For each participant, the training section consists of sending fNIRS examples to a machine learning model on known values, or in this case, movies that we already know the participant likes or dislikes. Instead of using preference ratings entered by people, the testing section uses machine learning classifiers to predict preference in real-time, which is used to provide updated movie recommendations to the user.

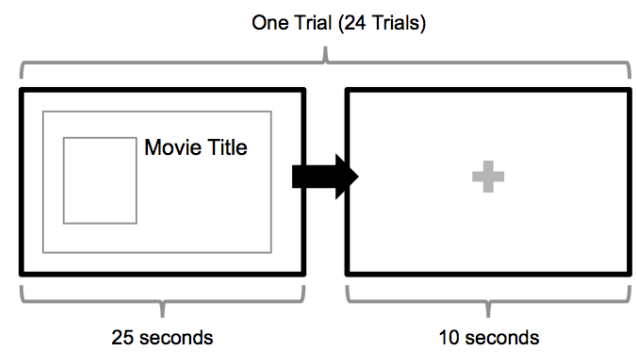

Figure 4: During training, participants viewed screenshots of their most favorite and least favorite movies for 25 seconds, followed by a 10 second rest period.

\section{$5.1 \quad$ Training}

At the start of the experiment, we provided participants with a list of movies picked from IMDB's list of 250 best movies and 100 worst movies and asked them to select their top three and bottom three. Participants viewed a timed slide show of selected movie webpages during which we recorded their brain activity with fNIRS. We showed each movie webpage for 25 seconds, followed by a rest period of 10 seconds (Figure 4). Participants viewed 12 slides of their top 3 movie titles and 12 slides of their bottom 3 movie titles. The brain activity recorded during these slides were used as training examples to our preference model. At the completion of training, the model was not altered for the remainder of the experiment. 

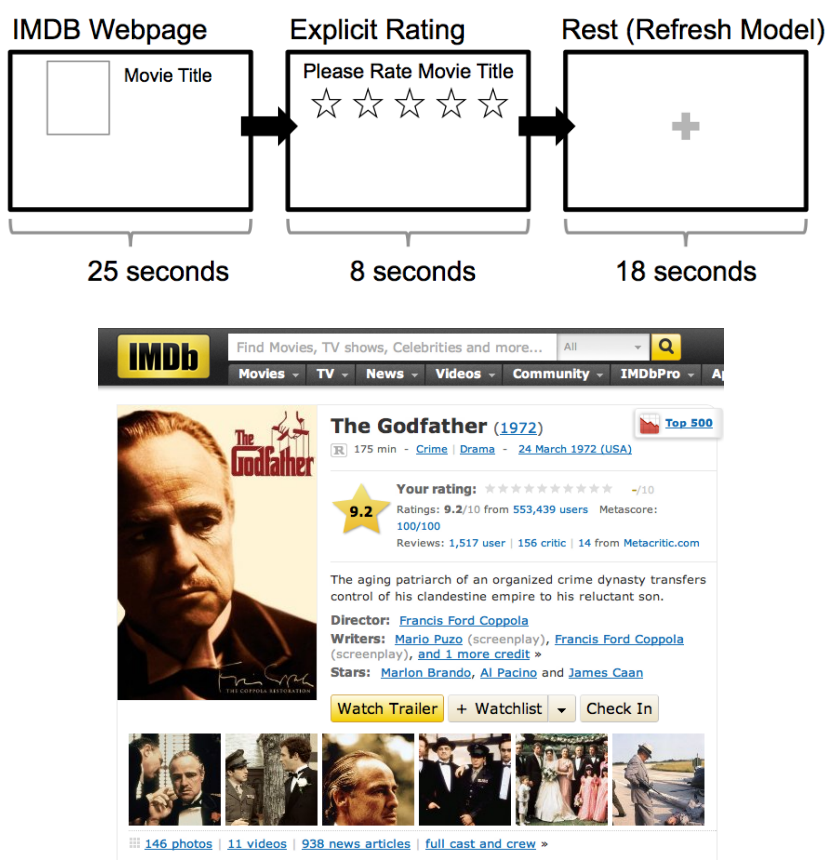

Figure 5: (Top) For each condition, participants viewed 20 movies. Each movie consisted of 25 seconds of viewing an IMDB page, an 8 second rating period, and an 18 second rest period. (Bottom) An example of an IMDB webpage participants viewed

\subsection{Testing}

In the testing section, participants viewed two trials, each of which consisted of a string of twenty movie websites, viewed sequentially. For each movie, participants viewed an IMDB page for 25 seconds, followed by an 8 second explicit rating period, and an 18 second rest period that enabled us to refresh the recommendation model (Figure 5).

Our motivation for exploring fNIRS is that it provides an implicit, unique signal from the user that is not accessible by other physiological sensors. Because of this, we believe that the first step in assessing its value is to compare it against a no-input environment. Thus, users interacted with two trials - one involving the brain recommendation system and one of a no input control condition.

In the control condition, a series of pre-defined movies with average ratings are used for all participants. This serves as a baseline for a no-input recommender. In the brain recommender condition, implicit preference ratings, as predicted by our fNIRS data classifier, are fed into a movie recommendation engine. We show the same start movie as the control condition, but new movies are selected based on previous preference values. For example, the 3rd movie is based on recorded preferences for the 1st and 2 nd movies.

fNIRS sensors remained attached to the participants during the course of the entire experiment, giving no indication of the condition. Following each movie in both conditions, participants were asked to provide an explicit preference rating of the movie (1-5 stars). We used this rating to evaluate the performance of our system. Unlike the implicit fNIRS readings, the ratings did not influence future recommendations in any way.

\section{RESULTS}

We ran this study with 6 male and 8 female volunteers $(N=14)$, aged 19-28 with a mean age of 22 . The order of conditions was counterbalanced across all participants. Given our hypothesis, we identify three measures to explore the efficacy of fNIRS in driving our recommendation system.

1. Recommendation ratings by condition: How did participants rate movies in the brain recommender condition in comparison to the control condition?

2. Recommendations over time: A good recommender should improve over time as it constructs a more accurate picture of the user's likes and dislikes. Does the brain-driven recommender give better recommendations over time?

3. Classification accuracy: How well did our system guess the user's preference for a given movie?

\subsection{Recommendation Ratings by Condition}

The key finding is that the brain recommender provided higher-rated movies than the control condition as the experiment session progressed. This difference becomes statistically significant after the 13th movie in each session. We display the distribution of all ratings in each condition in Figure 6.

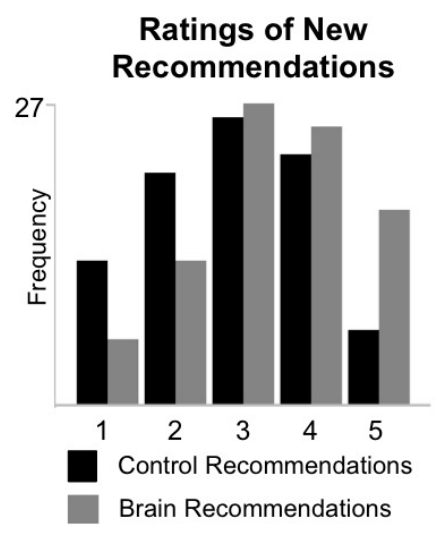

Figure 6: Histogram of ratings in the two conditions. We see that brain recommendations tend to be rated higher - mostly $3 \mathrm{~s}, 4 \mathrm{~s}$, and $5 \mathrm{~s}$.

We would not expect a recommendation system to perform well until it had seen enough examples to provide suitable recommendations. In our system, we saw this switch occur typically after the 13 th movie. We therefore analyzed the median rating in movies 14-20 for each participant across both conditions (see Table 1 for a summary). Running Mann-Whitney's U test on movies 14-20 revealed a significant effect of condition (the mean ranks of the control condition and brain recommendation condition were 10.46 and 18.54 , respectively; $U=41.5, Z=2.88, p<0.01, r=0.54$ ). 


\begin{tabular}{|l|c|c|c|}
\hline Condition & Median & Mean & Std. dev. \\
\hline Control & 3 & 2.9 & 1.17 \\
\hline Brain & 4 & 3.6 & 1.15 \\
\hline
\end{tabular}

Table 1: Ratings across movies 14 to 20

We also analyzed the median rating for the entire 20 movie session. As expected, we did not find a significant effect in condition (the mean ranks of the control condition and the brain condition were 11.75 and 17.25 , respectively; $U=$ $59.5, Z=1.86, p=.07, r=0.29)$.

To ensure the validity of these observations, we investigated whether our brain-computer recommender was aligning itself to individual preferences or simply gravitating to a small set of generally highly-rated movies. We found that 125 out of $280(45 \%)$ movie recommendations in the brain condition were unique selections, meaning that each participant saw an average of 9 movies no other participant viewed. These results support our primary hypothesis that the braindriven recommendation system recommended movies that catered to the participant's individual preferences.

\subsection{Recommendations Over Time}

Independent of the control condition, we find that recommendations from our system improved over time, suggesting that the preference model was gradually learning about the user. Across all participants, we analyzed the median rating given to movies at each time point (1-20) for each condition. For the brain recommender, we ran a linear regression and found that the total number of movies seen was a predictor of rating $(b=0.046, t(20)=$ $2.541, p=0.021)$. This means that over the course of 20 movies, the median recommendation improved by roughly one rating point (from 3 to 4 out of 5 ). The overall model fit was $R^{2}=0.223$. By comparison, applying a regression to the control condition determined that the number of movies seen did not predict movie rating $(b=0.004, t(20)=0.154, p=$ 0.898).

\subsection{Classification Accuracy}

Recall that we used a percentage vote from our classifiers to translate classifications of high and low preference into a 5 -point rating scale. To describe the accuracy of our system, we will use low preference to refer to ratings of 1 or 2 (out of 5) and high preference to refer to ratings of 4 or 5 .

In general, we found that our model skewed towards classifying movies as low preference (141 out of 280), while users tended to gravitate towards higher ratings. Figure 7 shows that when our model classified a movie as low preference, users were just as likely to have highly preferred the movie as they were to dislike it. However, when the model classified a movie as high preference (57 out of 280), users were five times more likely to give the movie a rating of 4 or 5 (out of 5) than 1 or 2 . This result is likely what drove the results from our system.

Taking a more fine-grained view of accuracy, the system precisely predicted the user's explicit preference rating in $27 \%$

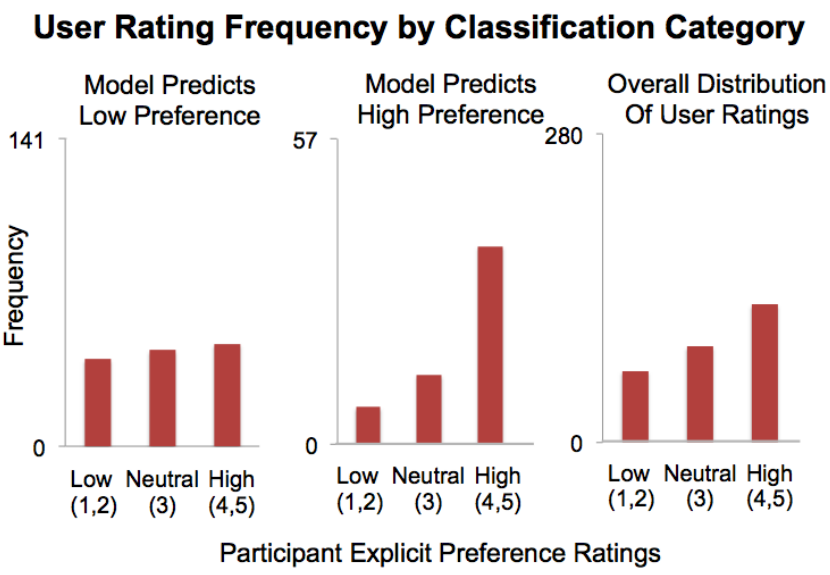

Figure 7: The preference model skewed towards classifying movies as low preference. However, when it classified the user's state as high preference, the user's explicit preference often agreed.

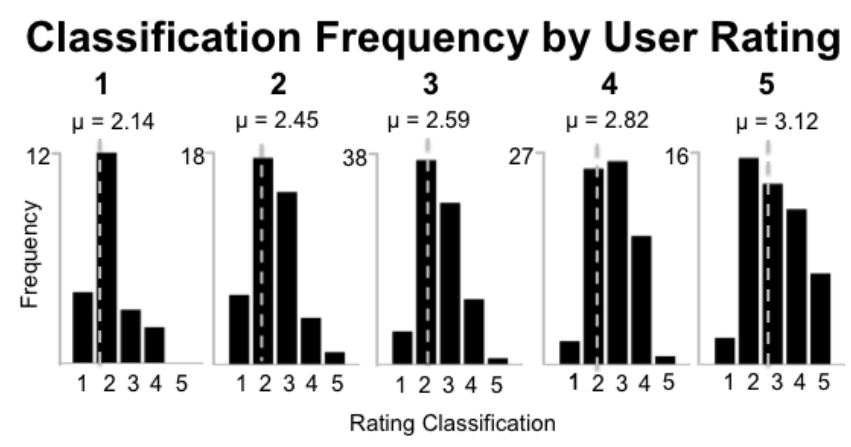

Figure 8: Frequency of predicted ratings for each user rating category (1-5). Although there was a wide variance of classifications for each rating category, relative to each other, the distributions of fNIRS classifications accurately mirror ratings by participants after each movie title.

of movies shown to the participant, and predicted within a single rating point for $72 \%$ of movies. Although the overall classification accuracy of the system indicates that improvements need to be made in signal processing or machine learning, we found that the mean prediction for each user rating (14 participants x 20 preference predictions) was accurate relative to each other rating category (see Figure 8 ).

\subsection{Anecdotal Evidence}

After completing the experiment, we asked participants which of the two batches of movies they preferred. Despite there being no detectable difference between conditions, 12 out of 14 participants immediately identified the group of movies recommended by the brain condition. In addition, several participants expressed regret for not recording movie titles that were recommended to them during the brain condition. 


\section{FNIRS AS INPUT AND FUTURE WORK}

In this paper, we presented a movie recommendation system that was driven by fNIRS input and performed better than a no-input recommender. We found that it provided unique recommendations across participants and that the preference model improved with increased interaction. These results suggest that fNIRS can be used as input to information filtering system.

Nonetheless, as we found in our experiment, misclassifications of user state are unavoidable. This is particularly true in early systems such as our own. Due to the necessary constraints imposed by user studies, we updated our model of user preference with each movie interaction, regardless of our confidence in the input. This lead to a reliance on classifications we knew were probably incorrect.

In a real system, we might ignore all classifications that fall beneath some confidence threshold, ensuring that the information we integrate into the user model is more likely to be reliable. For example, the performance of our preference classifier significantly increased when over $80 \%$ of our information channels classified the user as having high preference. If we build a system that exclusively relies on that information as input, we would expect to see more personalized recommendations and sharper increases in user satisfaction.

Moving forward, there are a number of active research areas that will serve to improve these classification rates: upgrading and increasing fNIRS sensors for better coverage of the prefrontal cortex, identifying information-rich channels on the probe, establishing features of the signal that best represent preference values, and improving training periods to discern optimal examples of user preference. With advances in machine learning and brain sensing technology we expect classifications of user state to increase in accuracy. In the meantime, designers must minimize the impact of misclassifications on the user.

\section{IMPLICATIONS}

Despite the current challenges of translating fNIRS input in real-time applications, we believe that brain sensing has the potential to positively influence the delivery of information to the user. We examine three ways in which fNIRS input may be employed in information filtering systems.

Because measurements of brain activity are largely an untapped source of information, performing better than a noinput control condition demonstrates that fNIRS can be used as an augmentative input in current recommendation systems. For example, one can envision Amazon or Netflix combining brain ratings with other implicit signals, such as purchase history and viewing history, to improve the overall accuracy of their model. Additionally, the user may be engaged in a high performance task where avoiding disruptions is critical. These implicit measures help preserve user attention because they do not force an externalization of subjective feelings onto a rating scale.

Moving away from movies and consumer products, we suggest that the true potential of neural measures lies outside current recommendation systems. In our study, participants viewed each movie website for a preset amount of time, and they were directed on a path of movies without an explicit option to diverge from it. While these measures were put in place to increase experimental control, they suggest that brain input may improve preference measures in domains where other implicit measures are difficult to obtain. For example, we can imagine a car radio station that naturally adapts its music to individual preferences without any intervention from the user.

Finally, we suggest that brain sensing may improve the comparison of information from disparate sources. Our work generalized Luu and Chau's measure of drink preference to movie preference. Thus, we do not expect dramatic changes in the physiological response to preference across disparate information sources. Using this general measure to redirect people towards relevant information both within and across websites could prevent disruption and save cognitive resources for primary working tasks.

\section{CONCLUSION}

In this work, we have shown that fNIRS brain sensing can be classified in real-time and applied to information filtering systems. Although there is still significant work before fNIRS can be translated to real-world environments, we suggest that brain-computer interfaces have the potential to aid users in everyday decisions and judgments as they continue to wrestle with an increasing quantity of information. In the past, researchers have identified periods of high workload in a number of scenarios using fNIRS. While that research highlighted the potential of BCIs recognizing when users may need information to be filtered, our work measuring preference begins to offer a solution for which information should be filtered or prioritized. Given these results, we believe that BCIs may one day provide user performance and satisfaction gains in an information saturated environment.

\section{ACKNOWLEDGMENTS}

We thank Beste Yuksel, Andrew Jenkins, Francine Lalooses, and Garth Griffin who are students in the HCI group at Tufts; Sergio Fantini and Angelo Sassaroli from the Biomedical Engineering Department at Tufts; and Erin Solovey from the Humans and Automation Lab at MIT. We thank the National Science Foundation (grant nos. IIS-0713506, IIS1065154, IIS-1218170) for support of this research.

\section{REFERENCES}

[1] H. Ayaz, P. A. Shewokis, S. Bunce, K. Izzetoglu, B. Willems, and B. Onaral. Optical brain monitoring for operator training and mental workload assessment. NeuroImage, 59(1):36-47, Jan. 2012.

[2] B. Bailey, J. Konstan, and J. Carlis. The effects of interruptions on task performance, annoyance, and anxiety in the user interface. In INTERACT, volume 1, 2001.

[3] B. P. Bailey and S. T. Iqbal. Understanding changes in mental workload during execution of goal-directed tasks and its application for interruption management. ACM Transactions on Computer-Human Interaction, 14(4):1-28, Jan. 2008.

[4] G. J. Barnum and C. A. Mattson. A Computationally Assisted Methodology for Preference-Guided Conceptual Design. Journal of Mechanical Design, 132(12):121003, 2010.

[5] A. Bechara, H. Damasio, A. R. Damasio, and G. P. Lee. Different contributions of the human amygdala 
and ventromedial prefrontal cortex to decision-making. The Journal of Neuroscience, 19(13):5473-81, July 1999.

[6] C. Calcanis, V. Callaghan, M. Gardner, and M. Walker. Towards end-user physiological profiling for video recommendation engines. Intelligent Environments, pages 1C2-1C2, 2008.

[7] I. Cantador, P. Brusilovsky, and T. Kuflik. Second workshop on information heterogeneity and fusion in recommender systems (HetRec2011). RecSys 2011, pages 387-388, 2011.

[8] M. Claypool, D. Brown, and P. Le. Inferring user interest. IEEE Internet Computing, 2001.

[9] E. Cutrell and D. Tan. BCI for passive input in HCI. In $A C M C H I$, volume 8, pages 1-3. Citeseer, 2007.

[10] M. Deppe, W. Schwindt, H. Kugel, and H. Nonlinear responses within the medial prefrontal cortex reveal when specific implicit information influences economic decision making. Journal of Neuroimaging, 15:171-182, 2005.

[11] S. H. Fairclough. Fundamentals of Physiological Computing. Interacting with Computers, 21:133-145, 2009.

[12] X. Fu, J. Budzik, and K. Hammond. Mining navigation history for recommendation. In $I U I$ ' 00 , New York, New York, USA, 2000. ACM.

[13] K. Gajos and D. S. Weld. Preference elicitation for interface optimization. UIST '05, 2005.

[14] L. George and A. Lécuyer. An overview of research on 'passive' brain-computer interfaces for implicit human-computer interaction. In International Conference on Applied Bionics and Biomechanics, 2010.

[15] A. Girouard, E. T. Solovey, L. M. Hirshfield, E. M. Peck, K. Chauncey, A. Sassaroli, S. Fantini, and R. J. Jacob. From Brain Signals to Adaptive Interfaces : Using fNIRS in HCI. In Brain-Computer Interfaces: Applying our Minds to Human-Computer Interaction. Springer, 2010.

[16] J. Gluck, A. Bunt, and J. Mcgrenere. Matching Attentional Draw with Utility in Interruption. In $\mathrm{CHI}$ '07, 2007.

[17] M. Hall, E. Frank, G. Holmes, and B. Pfahringer. The WEKA data mining software: an update. In $A C M$ SIGKDD, volume 11, pages 10-18, 2009.

[18] J. Healey, R. Picard, and F. Dabek. A New Affect-Perceiving Interface and Its Application to Personalized Music Selection The Affective DJ : Music Selection with the. Workshop on Perceptual User Interfaces, pages 2-5, 1998.

[19] L. M. Hirshfield, R. Gulotta, S. Hirshfield, S. Hincks, M. Russel, R. Ward, T. Williams, and R. J. K. Jacob. This is Your Brain on Interfaces : Enhancing Usability Testing with Functional Near-Infrared Spectroscopy. In $C H I$ ' $11,2011$.

[20] L. M. Hirshfield, E. T. Solovey, A. Girouard, J. Kebinger, R. J. K. Jacob, A. Sassaroli, and S. Fantini. Brain Measurement for Usability Testing and Adaptive Interfaces: An Example of Uncovering Syntactic Workload with Functional Near Infrared Spectroscopy. In $\mathrm{CHI}$ '09, 2009

[21] E. Horvitz and J. Apacible. Learning and reasoning about interruption. In Multimodal Interfaces '03, pages 20-27. ACM, 2003.

[22] J. Huber, D. Ariely, and G. Fischer. Expressing Preferences in a Principal-Agent Task: A Comparison of Choice, Rating and Matching. Organizational Behavior and Human Decision Processes, 87(1):66-90, 2002.

[23] S. Koelstra, A. Yazdani, M. Soleymani, C. Muhl, J. Lee, A. Nijholt, T. Pun, T. Ebrahimi, and I. Patras.
Single trial classification of EEG and peripheral physiological signals for recognition of emotions induced by music videos. Brain Informatics, pages 89-100, 2010.

[24] J. Kohlmorgen, G. Dornhege, M. Braun, B. Blankertz, K.-R. Muller, G. Curio, K. Hagemann, A. Bruns, M. Schrauf, and W. E. Kincses. Improving human performance in a real operating environment through real-time mental workload detection. In Toward Brain-Computer Interfacing, pages 409-422. MIT Press, 2007.

[25] S. Luu and T. Chau. Decoding subjective preference from single-trial near-infrared spectroscopy signals. Journal of Neural Engineering, 6, 2008.

[26] G. Mark and D. Gudith. The cost of interrupted work: more speed and stress. In CHI '08, pages 8-11, 2008.

[27] M. Marx and C. Schmandt. CLUES: dynamic personalized message filtering. In $C S C W$ ' 96 . ACM, 1996.

[28] S. M. McClure, J. Li, D. Tomlin, K. S. Cypert, L. M Montague, and P. R. Montague. Neural correlates of behavioral preference for culturally familiar drinks. Neuron, 44(2):379-87, Oct. 2004.

[29] M. Morita and Y. Shinoda. Information filtering based on user behavior analysis and best match text retrieval. In SIGIR '94, 1994.

[30] D. Nichols. Implicit rating and filtering. In DELOS Workshop on Filtering and Collaborative Filtering. Citeseer, 1997.

[31] J. Parsons, P. Ralph, and K. Gallagher. Using viewing time to infer user preference in recommender systems. In AAAI Workshop on Semantic Web Personalization, 2004.

[32] M. P. Paulus and L. R. Frank. Ventromedial prefrontal cortex activation is critical for preference judgments. Neuroreport, 14(10):1311-5, July 2003.

[33] H. A. Simon. Designing Organizations for an Information-Rich World. Computers, Communications, and the Public Interest, pages 37-72, 1971.

[34] E. Solovey, P. Schermerhorn, M. Scheutz, A. Sassaroli, S. Fantini, and R. Jacob. Brainput: Enhancing Interactive Systems with Streaming fNIRS Brain Input. In $C H I$ '12. ACM Press, 2012.

[35] E. T. Solovey, A. Girouard, K. Chauncey, L. M. Hirshfield, A. Sassaroli, F. Zheng, S. Fantini, and R. J. K. Jacob. Using fNIRS Brain Sensing in Realistic HCI Settings: Experiments and Guidelines. In UIST' $09,2009$.

[36] G. Strangman, J. P. Culver, J. H. Thompson, and D. A. Boas. A Quantitative Comparison of Simultaneous BOLD fMRI and NIRS Recordings during Functional Brain Activation. NeuroImage, 17(2):719-731, Oct. 2002.

[37] J. Teevan, S. Dumais, and E. Horvitz. Characterizing the value of personalizing search. In Proc. of Research and Development in Information Retrieval $200 \%$. ACM, 2007.

[38] A. Villringer and B. Chance. Non-invasive optical spectroscopy and imaging of human brain function. Trends in Neurosciences, 20(10):435-42, Oct. 1997.

[39] T.-L. Wu, Y.-P. Hung, Y.-Y. Chuang, H.-H. Chen, H. H. Chen, J.-H. Chen, S.-K. Jeng, H.-K. Wang, C.-C. Ho, Y.-P. Lin, T.-T. Hu, M.-F. Weng, L.-W. Chan, C.-H. Yang, and Y.-H. Yang. Interactive content presentation based on expressed emotion and physiological feedback. ACM Multimedia, 2008.

[40] T. Zander, C. Kothe, S. Jatzev, and M. Gaertner. Enhancing human-computer interaction with input from active and passive brain-computer interfaces. Brain-Computer Interfaces, pages 181-199, 2010. 